\title{
The Exoplanet Climate Infrared TElescope (EXCITE)
}

Gregory S. Tucker, Peter Nagler, Nathaniel Butler, Brian Kilpatrick, Andrei Korotkov, et al.

Gregory S. Tucker, Peter Nagler, Nathaniel Butler, Brian Kilpatrick, Andrei Korotkov, Nikole Lewis, Pierre F. L. Maxted, Laddawan Miko, C. B. Netterfield, Enzo Pascale, Jennifer Patience, Paul Scowen, Vivien Parmentier, Ingo Waldmann, Yiting Wen, "The Exoplanet Climate Infrared TElescope (EXCITE)," Proc. SPIE 10702, Ground-based and Airborne Instrumentation for Astronomy VII, 107025G (29 July 2018); doi: $10.1117 / 12.2314225$

Event: SPIE Astronomical Telescopes + Instrumentation, 2018, Austin, Texas, United States 


\title{
The Exoplanet Climate Infrared TElescope (EXCITE)
}

\author{
Gregory S. Tucker ${ }^{\mathrm{a}}$, Peter Nagler ${ }^{\mathrm{b}}$, Nathaniel Butler ${ }^{\mathrm{c}}$, Brian Kilpatrick ${ }^{\mathrm{a}}$, Andrei Korotkov ${ }^{\mathrm{a}}$, \\ Nikole Lewis ${ }^{\mathrm{d}}$, Pierre F. L. Maxted ${ }^{\mathrm{e}}$, Laddawan Miko ${ }^{\mathrm{b}}$, C. B. Netterfield ${ }^{\mathrm{f}}$, Enzo Pascale ${ }^{\mathrm{g}}$, \\ Jennifer Patience ${ }^{\mathrm{c}}$, Paul Scowen ${ }^{\mathrm{c}}$, Vivien Parmentier ${ }^{\mathrm{h}}$, Ingo Waldmann ${ }^{\mathrm{i}}$, and Yiting Wen ${ }^{\mathrm{b}}$ \\ ${ }^{a}$ Department of Physics, Brown University, Providence RI, USA \\ ${ }^{\mathrm{b}}$ NASA Goddard Space Flight Center, Greenbelt MD, USA

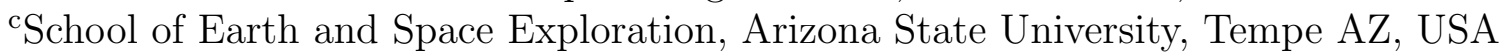 \\ ${ }^{\mathrm{d}}$ Space Telescope Science Institute, Baltimore MD, USA \\ ePhysics and Astrophysics, Keele University, Staffordshire, UK \\ ${ }^{\mathrm{f}}$ Department of Physics, University of Toronto, Toronto Ontario, Canada \\ gDepartment of Physics, La Sapienza Università di Roma, Rome, Italy \\ ${ }^{\mathrm{h}}$ Laboratoire d'Astrophysique de Marseille, Aix Marseille University, Marseille, France \\ ${ }^{i}$ Department of Physics and Astronomy, University College London, London, UK
}

\begin{abstract}
Although there are a large number of known exoplanets, there is little data on their global atmospheric properties. Phase-resolved spectroscopy of transiting planets - continuous spectroscopic observation of planets during their full orbits - probes varied depths and longitudes in the atmospheres thus measuring their three-dimensional thermal and chemical structure and contributing to our understanding of their global circulation. Planets with characteristics suitable for atmospheric characterization have orbits of several days, so phase curve observations are highly resource intensive, especially for shared use facilities. The Exoplanet Climate Infrared TElescope (EXCITE) is a balloon-borne near-infrared spectrometer designed to observe from 1 to $5 \mu \mathrm{m}$ to perform phaseresolved spectroscopy of hot Jupiters. Flying from a long duration balloon (LDB) platform, EXCITE will have the stability to continuously stare at targets for days at a time and the sensitivity to produce data of the quality and quantity needed to significantly advance our understanding of exoplanet atmospheres. We describe the EXCITE design and show results of analytic and numerical calculations of the instrument sensitivity. We show that an instrument like EXCITE will produce a wealth of quality data, both complementing and serving as a critical bridge between current and future space-based near infrared spectroscopic instruments.
\end{abstract}

Keywords: exoplanets, spectroscopy, phase curves, space instrumentation

\section{INTRODUCTION}

The Exoplanet Climate Infrared Telescope (EXCITE) is a balloon-borne spectrograph designed to perform phase-resolved spectroscopy of transiting hot Jupiters. EXCITE will measure spectroscopic phase curves of bright, short-period extrasolar giant planets (EGPs, or "hot Jupiters") near the peak of their spectral energy distributions (SEDs). The resulting phase-resolved spectroscopy will be used to map the temperature profile and chemical composition of planets as a function of planetary longitude. Combined with state-of-the-art threedimensional general circulation models (GCMs), these data will be used to study the atmospheric dynamics and chemistry in these strongly-irradiated planets. ${ }^{1}$

We still know very little about exoplanet atmospheric composition, thermal structure, and dynamics. ${ }^{2}$ Efforts to characterize exoplanet atmospheres have largely relied on space-based observatories such as the Spitzer Space Telescope and Hubble Space Telescope (HST), to remove the influence of Earth's atmosphere and achieve the highest levels of spectrophotometric precision. With moderate resolving power $(R \sim 50)$ and continuous spectroscopic coverage across wavelengths from 1-4 $\mu \mathrm{m}$, EXCITE will make observations that are inaccessible from

Further author information: (Send correspondence to Gregory S. Tucker)

Gregory S. Tucker: E-mail: gregory_tucker@brown.edu

Ground-based and Airborne Instrumentation for Astronomy VII, edited by Christopher J. Evans, Luc Simard, Hideki Takami, Proc. of SPIE Vol. 10702, 107025G · C 2018 SPIE · CCC code: 0277-786X/18/\$18 · doi: 10.1117/12.2314225 
existing observatories, helping to bridge the gap between current (e.g., HST) and future (e.g., JWST, WFIRST) space-based near infrared (NIR) spectroscopic observatories. EXCITE will operate from a high-altitude $(\sim 40$ $\mathrm{km}$ ) long duration balloon (LDB) platform, observing from a near-space environment above $>99 \%$ of the Earth's atmosphere. At these altitudes the Earth's atmosphere is stable and nearly transparent. This reduces the impact of systematic effects due to atmospheric variations that can limit lower-altitude experiments (see Figure 1). EXCITE's $0.5 \mathrm{~m}$ optical telescope assembly (OTA) is based on the successful Balloon-borne Imaging Testbed (BIT) platform. ${ }^{3}$ The pointing accuracy and stability of the BIT platform, combined with the circumpolar orbit of an Antartic LDB flight, allows EXCITE to continuously stare at targets through the duration of their orbits (up to several days). Such observations are resource-intensive for shared-use facilities, making them well suited for a purpose-built platform.

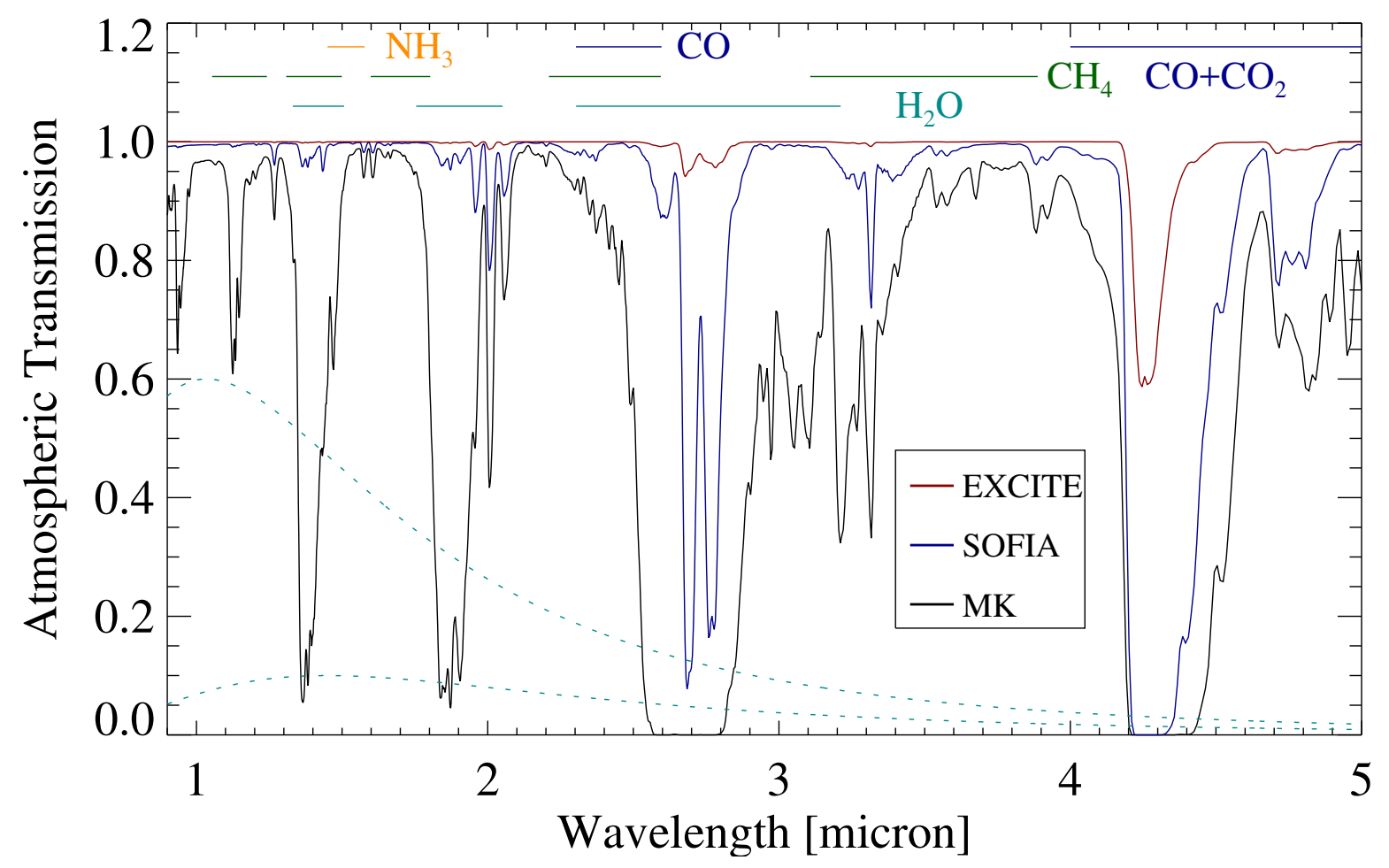

Figure 1. Atmospheric transmission for EXCITE, SOFIA and Mauna Kea (MK). Wavelengths of key molecular features for exoplanet atmosphere studies are indicated. Dotted lines are arbitrarily-scaled black body spectra at $1,800 \mathrm{~K}$ and $2,800 \mathrm{~K}$, i.e., typical dayside temperatures for our targets. The advantage of measuring exoplanet spectra from above the Earths atmosphere is clear.

\section{INSTRUMENT}

EXCITE uses a Ritchey-Chrétien telescope, nominally the same as the one used successfully in the test flight of the Balloon-borne Imaging Testbed (BIT). ${ }^{3}$ Since EXCITE, unlike BIT, is not an imaging instrument, distortionfree images across the focal plane are not required, so image corrector lenses are not needed. Compared to aplanatic Gregorian telescopes, Ritchey-Chrétien configurations are more compact and use smaller secondary mirrors, allowing more light to be collected for a given primary mirror size, better suiting the constraints of a balloon platform. The telescope structure is made from materials with similar coefficients of thermal expansion to ensure optical stability.

The basic optical layout of the spectrometer is shown in Figure 2. All the optics inside the cryostat are cooled to $77 \mathrm{~K}$. After entering the cryostat, the light from the telescope is divided into two bands $(1-2 \mu \mathrm{m}$ and $2-5 \mu \mathrm{m})$ using a dichroic. The light in each channel is focused on bandpass-optimized slits designed to limit 
the sky and instrument thermal background levels expected in a LDB flight to well below the expected source levels. After the slits, each beam passes through an Offner relay, with a prism positioned after the first concave mirror. In channel 1, the prism is made of Schott P-SF68 glass, which is a dense flint glass with a high index of refraction and high transmissivity $(T \geq 95 \%)$ in the $1-2 \mu \mathrm{m}$ band. Dense flint glasses are commonly used for NIR instrumentation and are suitable for cryogenic applications. ${ }^{4}$ In channel 2, the prism is made of sapphire, which performs well in cryogenic environments and has a transmissivity $T>85 \%$ from $1-4 \mu \mathrm{m}$. The transmissivity of sapphire is reduced at wavelengths longer than $4 \mu \mathrm{m}$, but there the EXCITE sensitivity is already limited by sky and telescope emission. The spectrometer configuration allows Nyquist sampling of the point spread function (PSF). Background spectra (within the slits) are obtained along the slit direction. overall optical efficiency is greater than $60 \%$. With near-diffraction-limited performance over the bandpass, the mean spectral resolution (Rayleigh criteria) is $\lambda / \Delta \lambda=50$. The focal plane detector is a Teledyne HAWAII-1RG (H1RG). The detector is maintained at an operating temperature just above $77 \mathrm{~K}$, where the median dark current level is $\leq 0.5 \mathrm{e}^{-} / \mathrm{s}$ per pixel. ${ }^{5}$ An internal calibrator similar to the one that will be used for ARIEL is used to monitor the performance of the detector array over time, correcting for detector system gain variations. ${ }^{6,7}$

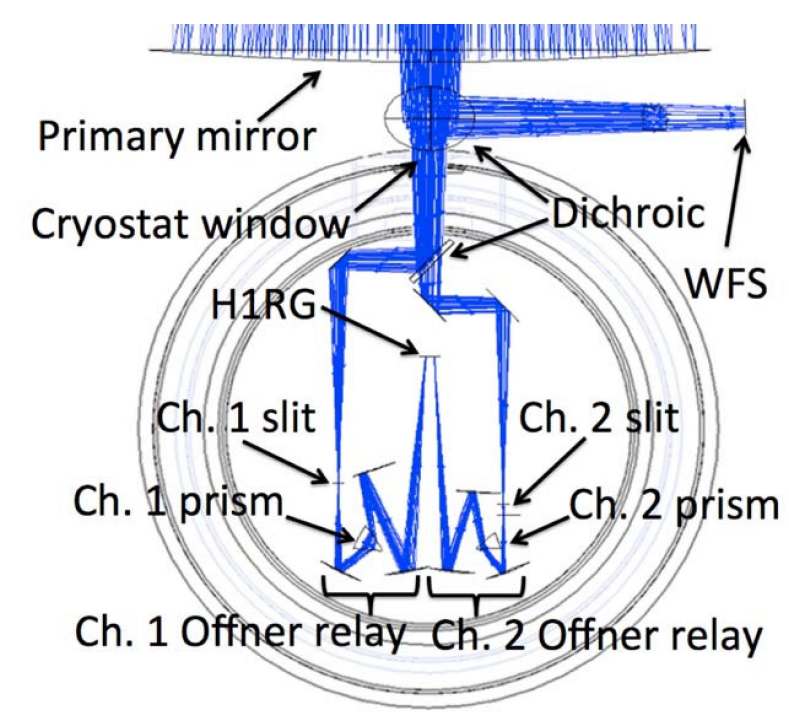

Figure 2. The optical layout of the spectrometer in the cryostat.

The EXCITE gondola and pointing system are based on the BIT platform, which was successfully demonstrated in a 2015 flight and is scheduled for a ultra long duration balloon (ULDB) flight from New Zealand in 2018. ${ }^{3}$ From the 8 hour flight, sub-arcsecond pointing stabilization of the telescope frame was demonstrated over tracking periods of more than an hour at a time. During a given tracking period, the telescope was stabilized to approximately 0.05 " rms. ${ }^{3}$ The EXCITE gondola (Figure 3 ) is designed to hold the $0.5 \mathrm{~m}$ diameter telescope in three axes with a peak-to-peak accuracy of better than 2 arcsec.

\section{SENSITIVITY}

Sky and telescope emission begin to dominate at $\lambda>4 \mu \mathrm{m}$, so we take $4 \mu \mathrm{m}$ as our longest science wavelength. We summarize the results of a radiometric sensitivity calculation in Figure 4. The figure shows the expected sensitivity per spectral bin in one hour of integration for the sources at the faint and bright ends of typical sources. These results assume uncorrelated noise from instrumental emission, the Earth's atmosphere, the target star, and detector dark current and readout.

\section{CORRELATED SYSTEMATIC EFFECTS}

To account for other noise sources, we simulated EXCITE observations of eclipses and phase curves. The simulator used for EXCITE is based on the time-domain simulator ExoSim. ${ }^{8}$ The simulations account for 

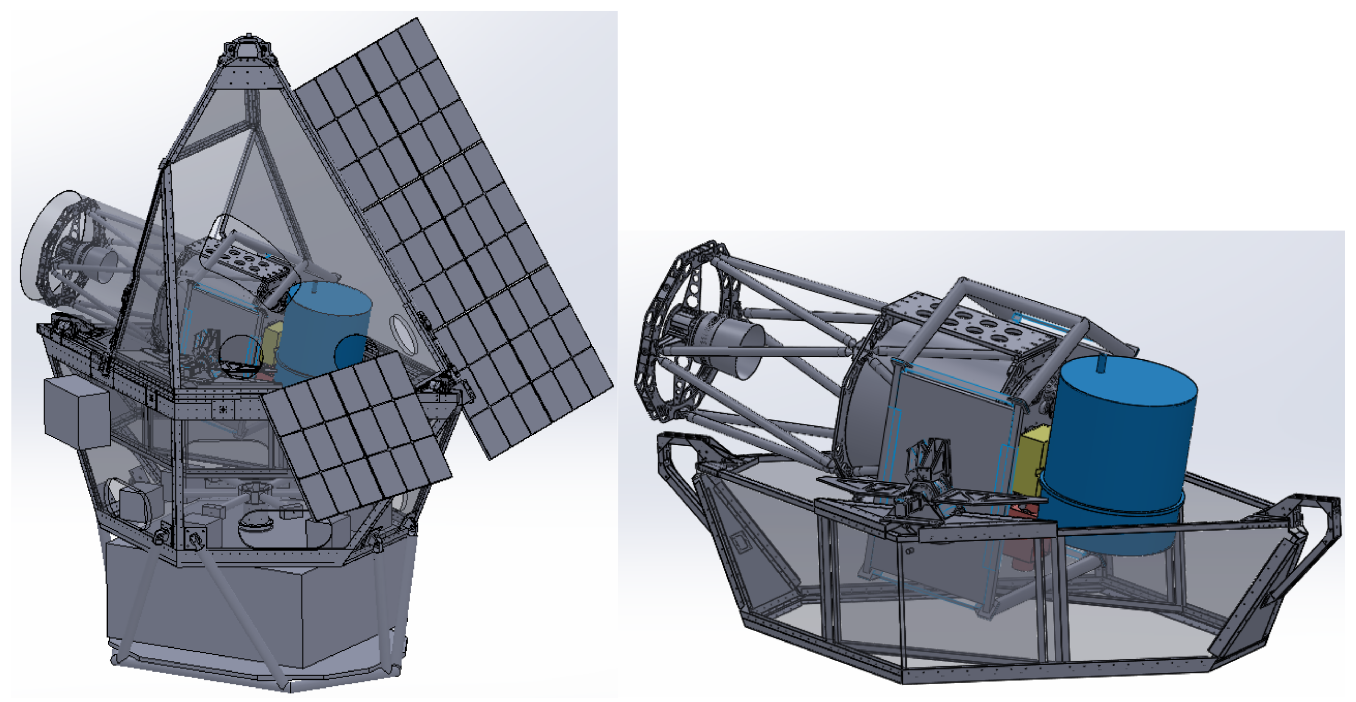

Figure 3. The EXCITE instrument. The telescope is a Ritchey-Chrétien design with a 0.5 m diameter primary mirror, which is pointed to an accuracy of 2 arcsec in all three axes. A fine guidance tip/tilt mirror provides the required 0.1 arcsec pointing stability. The telescope is surrounded by a temperature stabilized shroud. BIT successfully used such a shroud stabilized to $\pm 100 \mathrm{mK}$. The temperature is controlled by electrical heater pads.
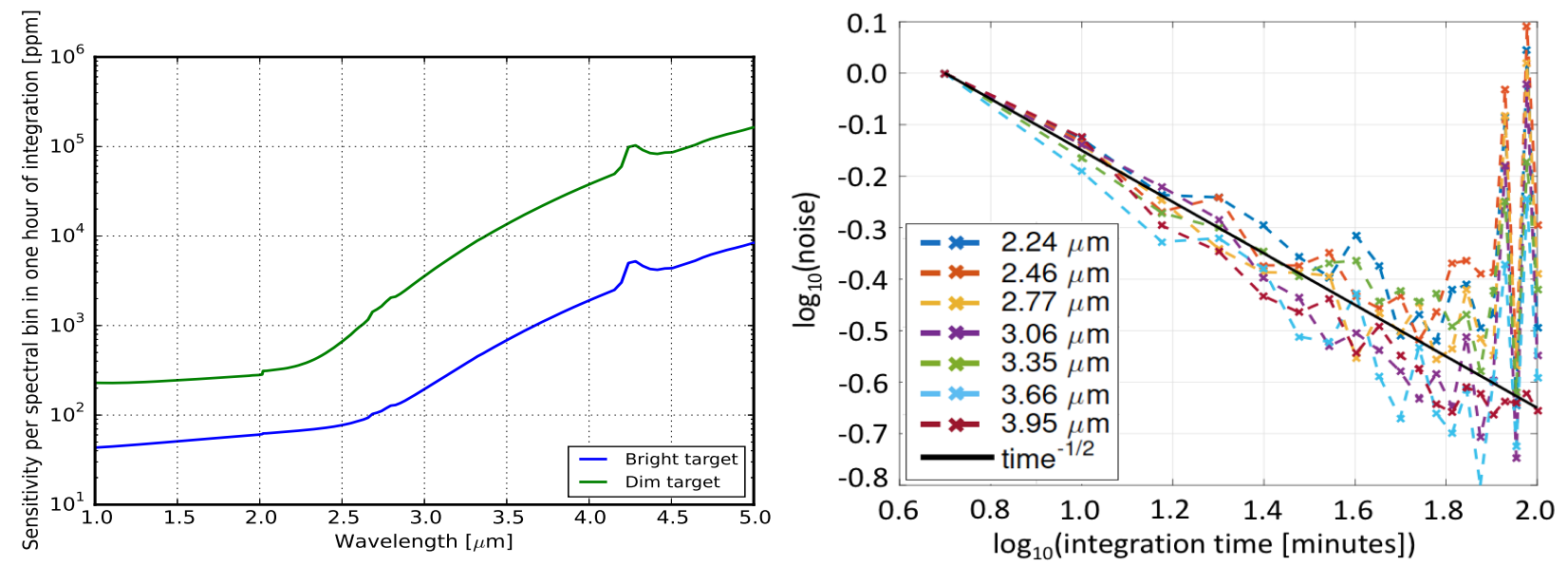

Figure 4. Left: Instrument sensitivity per spectral bin at $R=50$ for the faintest and brightest sources in one hour of integration. Because the PSF is Nyquist sampled, we are free to re-bin both spectrally and temporally to achieve better sensitivity. Right: Results of the EXCITE end-to-end simulation for an observation of the phase curve of WASP-18b. We show measurement noise across channel 2 as a function of integration time (channel 1, with higher SNR, is even more immune to correlated effects). Correlated effects do not contribute to the noise for measurements shorter than $\sim 90$ minutes.

uncorrelated shot noise and realistic time- and wavelength-correlated detector variation, pointing jitter, balloon altitude variations, and long-term instrumental drifts. In the following we discuss each of these sources, their impact on EXCITE's photometric stability and the mitigation strategies we will implement at the instrument level and in data analysis. The results of the simulation are shown in Figure 4, and indicate that the instrument is photon-noise limited for all reasonable integration times.

\subsection{Pointing jitter}

Pointing jitter is a source of photometric uncertainty arising from the motion of the sampled spectrum in both the spatial and spectral directions on the focal plane. Simulations of pointing jitter of 0.6 pixels RMS (significantly 
larger than the performance of the fine pointing control) show that post-processing jitter-induced systematics in the cross-dispersion direction are at least an order of magnitude below the photon noise. This remains true provided that i) the instrument monochromatic PSF is sampled by at least 2 detector pixels per FWHM (i.e., Nyquist sampled) and ii) the array can be flat-fielded to $\sim 0.5 \%$. Jitter-induced photometric systematics along the dispersion direction can be removed efficiently in data analysis using a combination of information from the attitude control system, the high SNR sampled stellar spectrum and decorrelation techniques, information about pixel wavelength response from pre-flight characterization, and in-flight calibration from the internal calibrator. ${ }^{7}$ Nyquist sampling the PSF avoids the most important correlated systematic effects in Spitzer ${ }^{9-11}$ arising from intra-pixel sensitivity variations. Moreover, the EXCITE detectors are similar to that of $H S T / \mathrm{WFC} 3$, which has negligible intra-pixel systematics. ${ }^{12}$

Using the calibration source EXCITE can achieve flat field accuracy of $0.5 \%$ - required to decorrelate jitter noise. Additional verification is achieved by observing sky background emission during ascent and at float and the internal calibrator at float. The Spitzer mission was able to achieve flat field accuracy of $\sim 0.1 \%{ }^{13}$

The slits widths are dimensioned to be the same size as the Airy disk at the red-end of each channel. The largest slit loss is $0.5 \%$ at $2 \mu \mathrm{m}$, assuming a worst case positioning of the PSF onto the slit to within $1 / 10$ th of the FWHM at this wavelength. Channel 2 monochromatic PSFs are larger, and the effect will be smaller. The slit design employed by EXCITE is similar to that of the ARIEL IR spectrometer, where slit losses were considered and the same conclusion was reached. ${ }^{7}$

\subsection{Atmospheric variations}

The Earth's atmosphere is a non-negligible diffuse background that changes as a function of elevation, altitude and Sun position. Similar variations occur in transmission, though at levels which are at all times subdominant to photon noise. This effect was modeled using MODTRAN. We account for two separate variations. On short timescales, the balloon's altitude oscillates with an amplitude of $\sim 50 \mathrm{~m}$ and a period of $\sim 5$ minutes, resulting in a $\sim 1.5 \%$ modulation of atmospheric emission across the EXCITE band. On longer timescales, the balloon altitude oscillates with an amplitude of $\sim 1 \mathrm{~km}$ and a period of $\sim 1$ day, modulating atmospheric emission by $\sim 30 \%$. Long-period elevation variations as a target is tracked also modulate sky emission, but at smaller levels than altitude variations. Integrating for longer than $\sim 5$ minutes removes short-period variations from the simulated time stream. This strategy is consistent with our observing plan, where the shortest observations are $\sim 1$ hour long. Diurnal variations contribute to the noise at a similar level to the uncorrelated sources; however this signal can be monitored and corrected by sampling detector pixels in the cross-dispersion direction.

\subsection{Instrumental drifts}

We simulated time variation of emission from the warm optical components by allowing the temperature of the warm optics to drift by a few degrees on $\sim 1$ day timescales. Noise due to temperature drifts is monitored and corrected in the same way as atmospheric emission.

Detector responsivity time variations are negligible $(<50 \mathrm{ppm})$ on few hour timescales. ${ }^{14,15}$ Possible longer timescale drifts will be corrected using a combination of stable G-type star observations and the calibrator. Detector latencies or persistence are well understood. It has been shown (e.g., the many studies involving WFC3 on HST) how these can be effectively dealt with using data analysis techniques (e.g., ${ }^{16}$ and references therein) and allowing sufficient settling time, which is built into our observing strategy. The detector is operated in a linear regime, and residual non-linear effects are negligible. A $5 \%$ divergence from linear response occurs at full well depth, ${ }^{17}$ and EXCITE will operate at $<75 \%$ well depth, similar to observations with HST $/$ WFC3, ${ }^{18}$ which employs the same detector technology.

\subsection{Stellar variability}

Stellar variability of the EXCITE targets at optical wavelengths due to star spots and stellar rotation has been characterized using the discovery light curves and is generally found to be $<0.1 \%$. Where variability is seen, the amplitudes at optical wavelengths are $\sim 1 \%$ and the rotation periods of the stars are found to be 10-20 days. Variability in the $1-5 \mu \mathrm{m}$ range is $3-4 \times$ less than at optical wavelengths for these solar-like stars ${ }^{19-21}$ and it is expected to have limited or negligible contribution to the noise budget in the IR. ${ }^{22,23}$ In general, the infrared 
variation is strongly correlated with the optical variation so monitoring of the star at optical wavelengths can be used to remove this noise source from the EXCITE spectroscopic data, as been demonstrated using Spitzer data. $^{24}$

\section{CONCLUSION}

A purpose-built instrument like EXCITE is best suited for resource-intensive phase curve measurements. Space missions like HST and JWST are multi-purpose. JWST will measure some phase curves, but given time allocation priorities it is unlikely to carry out as comprehensive a program as EXCITE. Not until a decade from now will ARIEL carry out such observations.

Unlike EXCITE, neither HST nor JWST can measure spectra of the brightest phase curve targets over the 1-4 $\mu \mathrm{m}$ band simultaneously. The HST NICMOS instrument has been used to measure exoplanet spectra up to $2.5 \mu \mathrm{m},{ }^{25-27}$ but it is not currently operational. The replacement for NICMOS, the WFC3, can observe only up to $1.7 \mu \mathrm{m} .{ }^{28} \mathrm{JWST}$ can access the entire $1-4 \mu \mathrm{m}$ band with the NIRSpec prism, but only for targets with a $\mathrm{J}$ magnitude fainter than $\approx 10,{ }^{29}$ thus excluding the targets best suited for phase-resolved spectroscopy. EXCITE will cover the entire 1-4 $\mu \mathrm{m}$ band, obtaining the signatures of water and carbon in a single orbital period observation.

With sensitivity comparable to that obtained by space-based observatories and sufficient bandwidth to measure key molecular features in the NIR, EXCITE will fill a key near-term need as a dedicated platform for studying exoplanet atmospheric physics.

\section{REFERENCES}

[1] Parmentier, V. and Crossfield, I. J. M., "Exoplanet Phase Curves: Observations and Theory," Handbook of Exoplanets, Edited by Hans J. Deeg and Juan Antonio Belmonte. Springer Living Reference Work, 116 (2017).

[2] Burrows, A. S., "Spectra as windows into exoplanet atmospheres," Proceedings of the National Academy of Science 111, 12601-12609 (Sept. 2014).

[3] Romualdez, L. J., Clark, P., Damaren, C. J., Galloway, M. N., Hartley, J. W., Li, L., Massey, R. J., and Netterfield, C. B., "Precise Pointing and Stabilization Performance for the Balloon-borne Imaging Testbed (BIT): 2015 Test Flight," ArXiv e-prints (Mar. 2016).

[4] Oliva, E. and Gennari, S., "Achromatic lens systems for near infrared instruments. II. Performances and limitations of standard Flint glasses," Astron. 83 Astrophys. Suppl. 128, 599-603 (Mar. 1998).

[5] Chuh, T., Loose, M., Gulbransen, D. J., Anglin, S. W., Beletic, J., Piquette, E. C., and Garnett, J. D., "Astronomy FPA advancements at Rockwell Scientific," Society of Photo-Optical Instrumentation Engineers (SPIE) Conference Series 6265, 2 (June 2006).

[6] EChO YellowBook, http://sci.esa.int/echo/53446-echo-yellow-book/.

[7] Ariel YellowBook, http://sci.esa.int/cosmic-vision/59109-ariel-assessment-study-report-yellow-book.

[8] Sarkar, S., Papageorgiou, A., and Pascale, E., "Exploring the potential of the ExoSim simulator for transit spectroscopy noise estimation," Proc. SPIE 9904, 99043R (July 2016).

[9] Ingalls, J. G., Krick, J. E., Carey, S. J., Laine, S., Surace, J. A., Glaccum, W. J., Grillmair, C. C., and Lowrance, P. J., "Intra-pixel gain variations and high-precision photometry with the Infrared Array Camera (IRAC)," Society of Photo-Optical Instrumentation Engineers (SPIE) Conference Series 8442, 1 (Sept. 2012).

[10] Deming, D., Knutson, H., Kammer, J., Fulton, B. J., Ingalls, J., Carey, S., Burrows, A., Fortney, J. J., Todorov, K., Agol, E., Cowan, N., Desert, J.-M., Fraine, J., Langton, J., Morley, C., and Showman, A. P., "Spitzer Secondary Eclipses of the Dense, Modestly-irradiated, Giant Exoplanet HAT-P-20b Using PixelLevel Decorrelation," ArXiv e-prints (Nov. 2014).

[11] Pont, F., Zucker, S., and Queloz, D., "The effect of red noise on planetary transit detection," Mon. Not. R. Astron. Soc. 373, 231-242 (Nov. 2006).

[12] Dressel, L., "Wide Field Camera 3 Instrument Handbook, Version 9.0 (Baltimore: STScI)," Handbook of Exoplanets, Edited by Hans J. Deeg and Juan Antonio Belmonte. Springer Living Reference Work (2017). 
[13] Spitzer Heritage Archive Documentation, IRAC Instrument and Instrument Support Teams, IRAC Instrument Handbook, 2.1 ed. (Feb. 2015).

[14] Clanton, C., Beichman, C., Vasisht, G., Smith, R., and Gaudi, B. S., "Precision Near-Infrared Photometry for Exoplanet Transit Observations. I. Ensemble Spot Photometry for an All-Sky Survey," Pub. Astron. Soc. Pac. 124, 700 (July 2012).

[15] Bezawada, N. and Ives, D., "High-speed multiple window readout of Hawaii-1RG detector for a radial velocity experiment," Proc. SPIE 6276, $62760 \mathrm{O}$ (June 2006).

[16] Tsiaras, A., Waldmann, I. P., Rocchetto, M., Varley, R., Morello, G., Damiano, M., and Tinetti, G., "A New Approach to Analyzing HST Spatial Scans: The Transmission Spectrum of HD 209458 b," ApJ 832, 202 (Dec. 2016).

[17] Blank, R., Anglin, S., Beletic, J. W., Baia, Y., Buck, S., Bhargava, S., Chen, J., Cooper, D., Eads, M., Farris, M., Hall, D. N. B., Hodapp, K. W., Lavelle, W., Loose, M., Luppino, G., Piquette, E., Ricardo, R., Sprafke, T., Starr, B., Xu, M., and Zandian, M., "The HxRG Family of High Performance Image Sensors for Astronomy," in [Solar Polarization 6], Kuhn, J. R., Harrington, D. M., Lin, H., Berdyugina, S. V., Trujillo-Bueno, J., Keil, S. L., and Rimmele, T., eds., Astronomical Society of the Pacific Conference Series 437, 383 (Apr. 2011).

[18] Berta, Z. K., Charbonneau, D., Désert, J.-M., Miller-Ricci Kempton, E., McCullough, P. R., Burke, C. J., Fortney, J. J., Irwin, J., Nutzman, P., and Homeier, D., "The Flat Transmission Spectrum of the SuperEarth GJ1214b from Wide Field Camera 3 on the Hubble Space Telescope," ApJ 747, 35 (Mar. 2012).

[19] Eddy, J. A., "The Sun, the Earth and Near-Earth Space: A Guide to the Sun-Earth System," ISBN 978-0-16-08308-8, 301 (2009).

[20] Knutson, H. A., Charbonneau, D., Allen, L. E., Fortney, J. J., Agol, E., Cowan, N. B., Showman, A. P., Cooper, C. S., and Megeath, S. T., "A map of the day-night contrast of the extrasolar planet HD 189733b," Nature 447, 183-186 (May 2007).

[21] Berta, Z. K., Charbonneau, D., Bean, J., Irwin, J., Burke, C. J., Désert, J.-M., Nutzman, P., and Falco, E. E., "The GJ1214 Super-Earth System: Stellar Variability, New Transits, and a Search for Additional Planets," ApJ 736, 12 (July 2011).

[22] Kreidberg, L., Line, M. R., Bean, J. L., Stevenson, K. B., Désert, J.-M., Madhusudhan, N., Fortney, J. J., Barstow, J. K., Henry, G. W., Williamson, M. H., and Showman, A. P., "A Detection of Water in the Transmission Spectrum of the Hot Jupiter WASP-12b and Implications for Its Atmospheric Composition," ApJ 814, 66 (Nov. 2015).

[23] Zellem, R. T., Swain, M. R., Roudier, G., Shkolnik, E. L., Creech-Eakman, M. J., Ciardi, D. R., Line, M. R., Iyer, A. R., Bryden, G., Llama, J., and Fahy, K. A., "Forecasting the Impact of Stellar Activity on Transiting Exoplanet Spectra," ApJ 844, 27 (July 2017).

[24] Knutson, H. A., Lewis, N., Fortney, J. J., Burrows, A., Showman, A. P., Cowan, N. B., Agol, E., Aigrain, S., Charbonneau, D., Deming, D., Désert, J.-M., Henry, G. W., Langton, J., and Laughlin, G., "3.6 and $4.5 \mu \mathrm{m}$ Phase Curves and Evidence for Non-equilibrium Chemistry in the Atmosphere of Extrasolar Planet HD 189733b," ApJ 754, 22 (July 2012).

[25] Crouzet, N., McCullough, P. R., Burke, C., and Long, D., "Transmission Spectroscopy of Exoplanet XO-2b Observed with Hubble Space Telescope NICMOS," ApJ 761, 7 (Dec. 2012).

[26] de Kok, R. J., Brogi, M., Snellen, I. A. G., Birkby, J., Albrecht, S., and de Mooij, E. J. W., "Detection of carbon monoxide in the high-resolution day-side spectrum of the exoplanet HD 189733b," Astron. \& Astrophys. 554, A82 (June 2013).

[27] Swain, M. R., Line, M. R., and Deroo, P., "On the Detection of Molecules in the Atmosphere of HD 189733b Using HST NICMOS Transmission Spectroscopy," ApJ 784, 133 (Apr. 2014).

[28] Stevenson, K. B., Désert, J.-M., Line, M. R., Bean, J. L., Fortney, J. J., Showman, A. P., Kataria, T., Kreidberg, L., McCullough, P. R., Henry, G. W., Charbonneau, D., Burrows, A., Seager, S., Madhusudhan, N., Williamson, M. H., and Homeier, D., "Thermal structure of an exoplanet atmosphere from phase-resolved emission spectroscopy," Science 346, 838-841 (Nov. 2014).

[29] STScI, "Space Telescope Science Institute, User Documentation for Cycle 1: Near Infrared Spectrograph Instrument (NIRSpec)." JWST User Documentation [Updated 12 February 2018] Baltimore, MD https: $/ /$ jwst-docs.stsci.edu. 